\title{
Chorea Associated with Diabetic Striatopathy in an Elderly Female: A Case Report
}

\author{
MR ISLAM ${ }^{\mathrm{a}}$, T RAHMAN ${ }^{\mathrm{b}}$, MSH KHAN $^{\mathrm{c}}$
}

\begin{abstract}
:
Diabetic striatopathy is a rare manifestation of diabetes mellitus. The disease commonly affects individuals of Asian descent, female, and the elderly. Patients usually present with hemiballism-hemichorea caused by hyperosmolar hyperglycemic state. Prompt recognition of a hyperglycemiainduced hemiballism-hemichorea is essential because the
\end{abstract}

\section{Introduction:}

Neurological manifestations of acute severe hyperglycemia are well described and the spectrum ranges from seizures to dyskinesias and coma. "Diabetic striatopathy" is an underdiagnosed complication of diabetes which is frequently encountered in T2DM than T1DM. It is a constellation of neurologic abnormalities defined by the dyskinesia of chorea-ballism and striatal abnormalities on neuroimaging. It has been usually described in elderly Asian females with hyperglycemic state and hyperosmolar state. The uniqueness of the presentation is that it is usually self-limiting with prompt management of the hyperglycemia. ${ }^{1}$ Here, we present a case of diabetic striatopathy who presented with chorea.

\section{Case summery:}

A 57-year-old right-handed woman with poorly controlled type 2 diabetes mellitus, hypertension and peripheral neuropathy presented to neurology OPD of

a. Dr. Md. Rashedul Islam, Assistant Professor, Department of Neurology, BIRDEM General Hospital, Dhaka, Bangladesh.

b. Dr. Tanbin Rahman, Registrar, Medicine, Greenlife Medical College and Hospital, Dhaka, Bangladesh.

c. Dr. Mohammad Sakhawat Hossen Khan, Registrar, Department of Neurology, BIRDEM General Hospital, Dhaka, Bangladesh.

Address of Correspondence: Dr. Md Rashedul Islam, Assistant Professor, Department of Neurology, BIRDEM General Hospital, Shahbag, Dhaka-1000, Phone: 01819216209, Email: rashed2k2001@yahoo.com

Received: 15 Sept., 2019

Accepted: 08 Sept., 2021 symptoms are reversible with correction of hyperglycemia. Diagnosis is possible based on blood reports and neuroimaging findings.

Keywords: Diabetic striatopathy, chorea

(J Bangladesh Coll Phys Surg 2022; 40: 65-67) DOI: https://doi.org/10.3329/jbcps.v40i1.57061

BIRDEM general hospital for choreiform movements of the right upper and lower extremities for the past 1 month. She noted no abnormal movements elsewhere in the body and had no neuropsychiatric history. The movements disappear during sleep. She has consulted several physicians and trials of trihexyphenidyl and carbidopa-levodopa were given without any effect.

She had no history of neuroleptic or antiemetic exposure. Her only medications were insulin, olmesartan and metoprolol. She was poorly compliant with the insulin. Family history was unremarkable. She is non smoker, non alcoholic.

General neurological examination revealed a normal higher psychic function including speech and cranial nerves are intact. On motor examination, there was nearly continuous low-amplitude choreiform movement of right upper and lower limb including tongue and face, without evidence of bradykinesia or rigidity. She had slightly reduced deep tendon reflexes and impaired vibration sensation, consistent with her known peripheral neuropathy. Gait formulation was impaired.

Investigations revealed initial plasma glucose of 594 $\mathrm{mg} / \mathrm{dL}$, with HbA1c of $15.2 \%$, serum sodium was 148 $\mathrm{mmol} / \mathrm{l}$, and calculated serum osmolality was $338 \mathrm{mosm} /$ $\mathrm{kg}$. Serum ketones were negative and the arterial blood gas analysis was normal. Renal function and liver function tests were normal. Non-contrast computed tomography (CT) of brain revealed hyper density in the left basal ganglia region (Fig 1). MRI brain showed T1 hyperintensity in the left globus pallidus and putamen 
with T2 hypointensity in the same area with sparing of the internal capsule consistent with diabetic striatopathy (Fig 2). With this clinical presentation and MRI findings, a diagnosis of diabetic striatopathy was made. Her diabetes was managed with insulin and she was put on low dose of haloperidol (2.5 $\mathrm{mg}$ twice daily) to control involuntary movements. Within 7 days she showed marked improvement in symptoms. The patient is doing well clinically and under regular follow up. No follow up brain imaging was done.

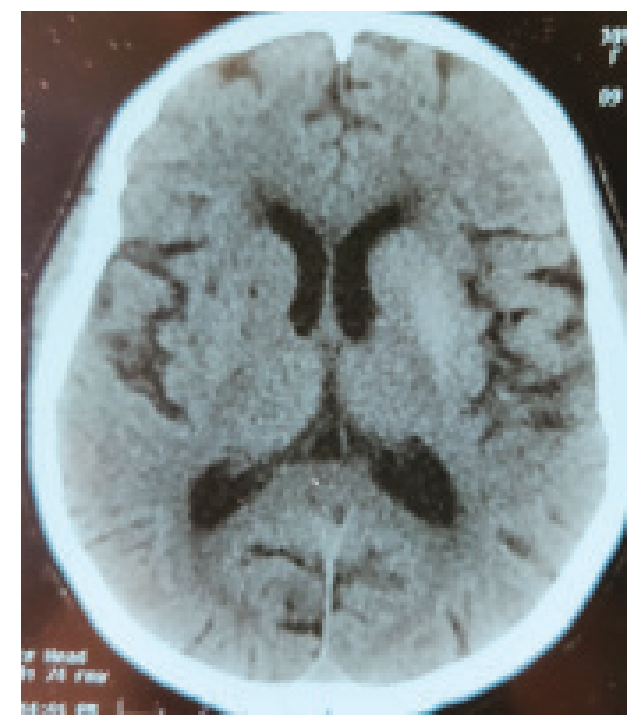

Fig.-1: (hyperdense lesion on left basal ganglia)

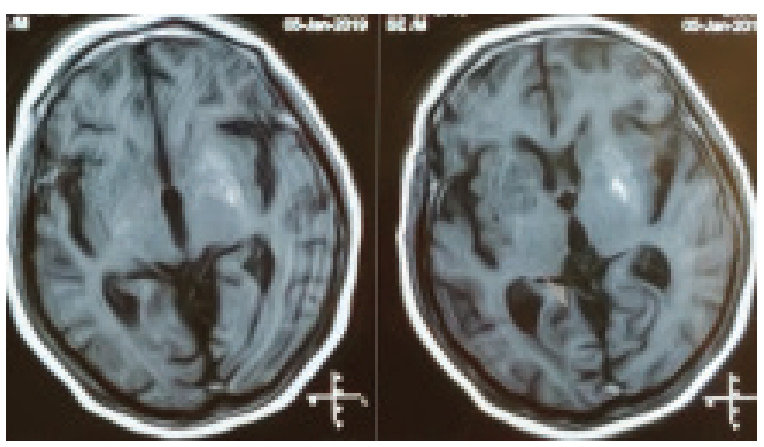

Fig.-2: (T1 hyperintensity in the left globus pallidus and putamen)

\section{Discussion:}

Hemichorea as a manifestation of nonketotic hyperglycemia $(\mathrm{NKH})$ is a rarely described entity. This is typically seen in elderly Asian women with type 2 diabetes, though it has also been reported in type 1 diabetes, those with diabetic ketoacidosis and in children. ${ }^{2,3}$ There is characteristic vulnerability of the striatum (caudate nucleus and putamen and/or globus pallidus) to the acute metabolic insult. It is characterized by high signal on T1-weighted MRI confined to the striatum. Pathophysiological mechanism in diabetic striatopathy involves hyperviscosity leading to local tissue hypoperfusion, depletion of gammaAminobutyric acid, and accumulation of manganesecontaining gemistocytes in the basal ganglia which typically appear as T1 hyperintense lesions. ${ }^{4}$ In a large meta-analysis of 53 cases, two-third of the patients had complete resolution of the symptoms during follow up after 3 months, one-third of them only with control of hyperglycemia. ${ }^{5}$ The high signal intensity T1-weighted lesion in basal ganglia may also be seen in hepatic encephalopathy, post-cardiac arrest encephalopathy, and hypoglycemic coma. ${ }^{5}$ Though generally having a benign outcome, this condition can lead to caudate atrophy and persistent movement disorder if not recognized and treated properly. ${ }^{6}$

During hyperglycemic crisis, the Kreb's cycle activity is suppressed in the brain, so cerebral metabolism shifts to alternative anaerobic pathways. In hyperosmolar hyperglycemic state, this shift causes the brain to metabolise gamma-Aminobutyric acid (GABA) into succinic acid via the semialdehyde pathway, leading to rapid depletion of GABA levels. ${ }^{7}$ Thus, its depletion causes disinhibition of the thalamus by the medial globus pallidus, resulting in hyperkinetic movements. On the other hand, in diabetic ketoacidosis acetoacetate produced from the liver is available to produce GABA thereby preventing its low levels, hence the rarity of striatopathy in DKA. ${ }^{8}$

Treatment usually involves correction of the metabolic derangement by fluids and insulin infusion. Additional drugs including neuroleptic agents (haloperidol/ risperidone), dopamine-depleting agents (reserpine/ tetrabenazine), GABAergic drugs and selective serotonin reuptake inhibitors like escitalopram are required sometimes. Complete clinical resolution, with or without radiologic resolution, is usually a feature. The time to resolution has been reported to be variable. ${ }^{1}$

A large case series demonstrated that patients had complete resolution of symptoms that were treated by controlling hyperglycemia and standard treatment of hyperkinetic movement. Follow-up imaging in some case reports suggested that, the T1 hyperintensity in MRI of 
brain resolves along with the movement symptoms as hyperglycemia is corrected. ${ }^{9}$ Our case had resolved within a week with haloperidol and other medical management. There was no specific correlation with initial blood glucose, duration of diabetes or serum osmolarity at time of admission although longer time is needed in cases of poorer diabetes control and elderly patients.

\section{Conclusion:}

Diabetic striatopathy due to hyperosmolar hyperglycemic state is a rare entity. Prompt recognition and treatment reduces morbidity and is rewarding.

\section{Conflict of interest: None}

\section{References:}

1. Liza Das, Rimesh Pal, Pinaki Dutta, Anil Bhansali. "Diabetic striatopathy" and ketoacidosis: Report of two cases and review of literature. Diabetes research and clinical practice. $2017 ; 128: 1-5$.

2. Faundez T, Klee P, Hanquinet S, Schwitzgebel V, Burkhard PR, Korff CM, et al. Diabetic striatopathy in childhood: A case report. Pediatrics. 2016;137 pii: e20143761.

3. Khan I, Kant C, Sanwaria A, Goyal A. Hemichoreahemiballismus as the presenting manifestation of nonketotic hyperglycemia in an adolescent with undiagnosed type 2 diabetes mellitus. Indian J Endocrinol Metab. 2012; 16(Suppl 1):S129-31.

4. Abe Y, Yamamoto T, Soeda T, Kumagai T, Tanno Y, Kubo J, et al. Diabetic striatal disease: Clinical presentation, neuroimaging, and pathology. Intern Med. 2009; 48: $1135-41$.

5. Oh SH, Lee KY, Im JH, Lee MS. Chorea associated with non-ketotic hyperglycemia and hyperintensity basal ganglia lesion on T1-weighted brain MRI study: A meta-analysis of 53 cases including four present cases. J Neurol Sci. 2002; 200:57-62.

6. Lucassen EB, Delfyett WT, Stahl MC. Persistent hemichorea and caudate atrophy in untreated diabetic striatopathy: A case report. Case Rep Neurol. 2017;9: 299-303.

7. Lin JJ, Chang MK. Hemiballism-hemichorea and nonketotic hyperglycaemia. J Neurol Neurosurg Psychiatry 1994; 57:748-50.

8. Mihai CM, Catrinoiu D, Stoicescu RM. Atypical onset of diabetes in a teenage girl: a case report. Cases J 2008 Dec $30 ; 1(1): 1$.

9. Oh SH, Lee KY, Im JH, Lee MS. Chorea associated with non-ketotic hyperglycemia and hyperintensity basal ganglia lesion on T1-weighted brain MRI study: a meta-analysis of 53 cases including four present cases. Journal of the Neurological Sciences, 01 Aug 2002, 200(1-2):57-62. 